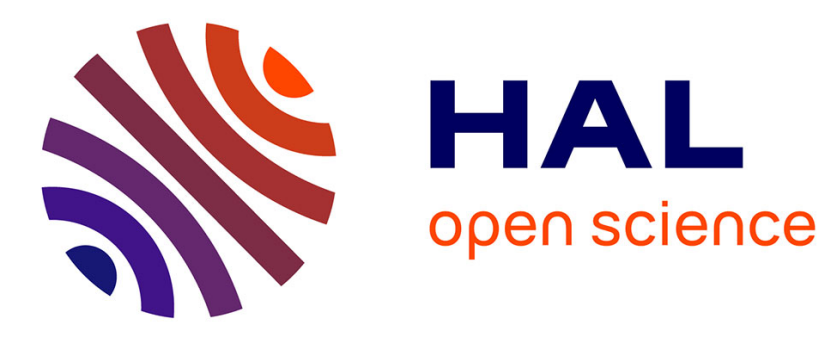

\title{
Carrier dynamics in Landau-quantized graphene featuring strong Auger scattering
}

Martin Mittendorff, Florian Wendler, Ermin Malic, Andreas Knorr, Milan

Orlita, Marek Potemski, Claire Berger, Walter A. de Heer, Harald Schneider, Manfred Helm, et al.

\section{To cite this version:}

Martin Mittendorff, Florian Wendler, Ermin Malic, Andreas Knorr, Milan Orlita, et al.. Carrier dynamics in Landau-quantized graphene featuring strong Auger scattering. Nature Physics, 2015, 11 (1), pp.75 - 81. 10.1038/nphys3164 . hal-01784086

\section{HAL Id: hal-01784086 \\ https://hal.science/hal-01784086}

Submitted on 3 May 2018

HAL is a multi-disciplinary open access archive for the deposit and dissemination of scientific research documents, whether they are published or not. The documents may come from teaching and research institutions in France or abroad, or from public or private research centers.
L'archive ouverte pluridisciplinaire HAL, est destinée au dépôt et à la diffusion de documents scientifiques de niveau recherche, publiés ou non, émanant des établissements d'enseignement et de recherche français ou étrangers, des laboratoires publics ou privés. 


\title{
Ultrafast carrier dynamics in Landau-quantized graphene: strong Auger scattering
}

\author{
Martin Mittendorff ${ }^{1,2}$, Florian Wendler ${ }^{3}$, Ermin Malic ${ }^{3}$, Andreas Knorr ${ }^{3}$, Milan Orlita ${ }^{4,5}$, \\ Marek Potemski ${ }^{4}$, Claire Berger ${ }^{6,7}$, Walter A. de Heer ${ }^{6}$, Harald Schneider ${ }^{1}$, Manfred \\ Helm $^{1,2}$, and Stephan Winnerl ${ }^{1}$ \\ ${ }^{1}$ Helmholtz-Zentrum Dresden-Rossendorf, P.O. Box 510119, 01314 Dresden, Germany \\ ${ }^{2}$ Technische Universität Dresden, 01062 Dresden, Germany \\ ${ }^{3}$ Technische Universität Berlin, Hardenbergstraße 3610623 Berlin, Germany \\ ${ }^{4}$ Laboratoire National des Champs Magnétiques Intenses, CNRS-UJF-UPS-INSA, 38042 \\ Grenoble, France \\ ${ }^{5}$ Charles University Faculty of Mathematics and Physics, Ke Karlovu 5, 12116 Praha, Czech \\ Republic \\ ${ }^{6}$ Georgia Institute of Technology, Georgia, Atlanta 30332, United States \\ ${ }^{7}$ CNRS - Institut Néel, 38042 Grenoble, France \\ Email: m.mittendorff@hzdr.de
}

\begin{abstract}
The energy spectrum of common two-dimensional electron gases consists of a harmonic, i.e. equidistant ladder of Landau levels, thus preventing the possibility to optically address individual transitions. In graphene, however, due to its non-harmonic spectrum, individual levels can be addressed selectively. We report here the first time-resolved experiment directly pumping discrete Landau levels in graphene. Energetically degenerate Landau-level transitions from $n=-1$ to $n=0$ and from $n=0$ to $n=1$ are distinguished by applying circularly polarized THz light. In agreement with our experimental results, an analysis based on microscopic theory shows that the zeroth Landau level is actually depleted by strong Auger scattering, even though it is optically pumped at the same time. Such a phenomenon has never been observed before in any system to our knowledge. The surprisingly strong electron-electron interaction responsible for this effect is directly evidenced through a sign reversal of the pump-probe signal.
\end{abstract}


Transport experiments on graphene in magnetic fields have revealed a number of fascinating phenomena such as quantum ratchet effects ${ }^{1}$, the Hofstadter butterfly ${ }^{2-4}$ and the fractional quantum Hall effect ${ }^{5,6}$. The anomalous quantum Hall effect, which is a consequence of a Landau level at zero energy in graphene (cf. Fig. 1a), is considered a hallmark of the Diracfermion nature of charge carriers in graphene ${ }^{7,8}$. The non-equidistant Landau-level (LL) structure has been observed in various continuous-wave (cw) magneto-spectroscopy experiments $^{9-12}$, which provided evidence for the Dirac-cone band structure at various energies $^{10,11}$. Furthermore this technique allowed one to determine the Fermi velocity ${ }^{9}$ and to identify graphene of extremely high quality ${ }^{12}$. The observation of a giant Faraday rotation of light passing through graphene in magnetic fields as well as the demonstration of tunable $\mathrm{THz}$ detectors highlight the application potential of graphene in magneto-optic devices ${ }^{13,14}$. For more sophisticated applications such as light emitters, however, a detailed knowledge of the carrier dynamics is required.

While the relaxation dynamics in graphene at zero magnetic field has been studied intensively ${ }^{15-20}$ there is so far only one time-resolved spectroscopy study in the presence of a magnetic field ${ }^{21}$. Plochocka and co-workers performed a pump-probe study at high energies corresponding to the excitation of a quasi-continuum of LLs characterized by a LL separation smaller than the LL broadening. The observed increase in relaxation time at enhanced magnetic fields was attributed to a suppression of Auger processes ${ }^{21}$. The role of Auger scattering bridging the valence and conduction bands and changing the number of charge carriers has been controversially discussed for graphene in the absence of magnetic fields ${ }^{19,22,23}$. Charge carriers fulfill energy and momentum conservation for Auger processes only along a straight line on the Dirac cone. It has been argued that the contribution of processes restricted to lines in $k$-space vanishes in the two-dimensional phase space. However, full quantum-mechanical treatments of the ultrafast carrier dynamics indicate that Auger 
processes, i.e. Auger recombination and impact ionization, are very efficient in graphene ${ }^{19,24-}$ 26. Impact ionization can result in carrier multiplication in graphene on sub-ps timescales, which has recently been verified experimentally ${ }^{19,20,27}$. Due to their specific energy structure, the energetically lowest Landau levels, which can be selectively excited in the experiment, open a unique route to investigate the importance of Auger scattering in graphene.

In this Article, we present a joint experiment-theory study investigating the carrier dynamics in Landau-quantized graphene by selectively pumping and probing transitions between lowenergy Landau levels. Hence, for the first time, the discrete graphene LL system is investigated in a time-resolved study. We apply radiation with a photon energy of $75 \mathrm{meV}$ (wavelength: $16.5 \mu \mathrm{m}$ ) and magnetic fields of up to $7 \mathrm{~T}$ to address the possible relaxation channels of excited electrons in the presence of a magnetic field. Thorough understanding of the dynamics in this unique discrete-level system is obtained by performing pump-probe experiments with circularly polarized radiation, which allows one to selectively address the degenerate $\mathrm{LL}_{-1} \rightarrow \mathrm{LL}_{0}$ and $\mathrm{LL}_{0} \rightarrow \mathrm{LL}_{1}$ transitions (cf. Fig. 1a). Our main observation is that for one of the four possible combinations of pumping and probing with left and rightcircularly polarized radiation, the differential transmission signal (DTS) shows the opposite sign with respect to the signal expected from the usually applied single particle absorption bleaching scheme. This observation reveals a highly efficient elastic relaxation channel that we can unambiguously identify by performing microscopic time-resolved calculations of the carrier dynamics. The experiments and calculations show that Auger scattering is the predominant relaxation process giving rise to a redistribution of carriers in Landau-quantized graphene on a ps timescale. The obtained fundamental insights into the nature of carriercarrier scattering in graphene in the presence of a magnetic field are relevant for novel applications, such as graphene-based Landau lasers. 
The time-resolved experiments were performed on multilayer ( 40 layers) epitaxial graphene (MEG) grown by thermal decomposition on the C-terminated face of $\mathrm{SiC}^{28}$. The decoupled nature of the layers of the sample was evidenced by Raman spectroscopy ${ }^{29}$ and cw magnetospectroscopy $^{9}$. The majority of graphene layers is quasi-intrinsic ${ }^{11,18}$, while the layers close to the interface of $\mathrm{SiC}$ exhibit a pronounced n-type doping ${ }^{16,30,31}$. The free-electron laser FELBE delivered radiation pulses with a duration of 2.7 ps (full width at half maximum, FWHM) and a photon energy of $75 \mathrm{meV}$. Various pump-probe experiments were performed involving linear polarization as well as left and right-circularly polarized radiation for pumping and probing. A simplified sketch of the experiment with linearly polarized radiation is shown in the inset of Fig. 1b. Details on the experimental configuration can be found in the methods section. In all experiments, the sample temperature was kept at $10 \mathrm{~K}$ in a cryostat that allows for applying magnetic fields up to $7 \mathrm{~T}$.

In the following, induced transmission transients (Fig. 1b) are analyzed. The features of the induced transmission amplitudes (Fig. 1d) and the initial relaxation time (Fig. 1e) are identified by comparing their B-field dependence to the dynamic conductivity of a graphene layer (Fig. 1c). The dynamic conductivity that is proportional to the absorption is calculated using the Kubo formalism ${ }^{9}$, considering the dipole radiation selection rule $|\Delta n|=1$. A width of $7 \mathrm{meV}$ is chosen in accord with $\mathrm{cw}$ magneto-spectroscopy data ${ }^{11}$. The calculation allows us to attribute peaks in the induced transmission to the interband transitions $\mathrm{LL}_{-1(0)} \rightarrow \mathrm{LL}_{0(1)}$, $\mathrm{LL}$. $2(-1) \rightarrow \mathrm{LL}_{1(2)}$, and $\mathrm{LL}_{-3(-2)} \rightarrow \mathrm{LL}_{2(3)}$ in quasi-intrinsic graphene layers (cf. Fig. 1c and 1d). Upon resonance with these transitions the induced transmission is enhanced. The strongest resonant enhancement (factor of 2.5 relative to the signal at $B=0$ ) is observed for the energetically lowest transition that exhibits the largest oscillator strength (cf. Fig. 1d). Note that due to the vanishing density of states between LLs (e.g. for $1 \mathrm{~T}<B<3 \mathrm{~T}$ ), the pump- 
probe signal is expected to vanish in this region. The observed non-zero signals in the experiment most likely stem from intraband LL transitions in the highly doped graphene layers close to the interface to the $\mathrm{SiC}$ substrate. In $\mathrm{cw}$ magneto-spectroscopy experiments, broad absorption features from doped layers have already been observed ${ }^{32,33}$ supporting this interpretation.

For $B=0$, the carrier relaxation dynamics is characterized by a single exponential decay with a time constant of $\tau=18 \pm 3$ ps (cf. Figs. $1 \mathrm{~b}$ and $1 \mathrm{e}$ ). In a previous study, this time constant was attributed to scattering via optical phonons ${ }^{18}$. For finite magnetic fields, a doubleexponential behavior is found (cf. Fig. 1b). The observed initial decay time $\tau$ slightly increases with the magnetic field up to $22 \pm 3$ ps for $B=3 \mathrm{~T}$ (cf. Fig. 1e). For $B=4.2 \mathrm{~T}$, the excitation energy is in resonance with the energetically lowest $\mathrm{LL}_{-1(0)} \rightarrow \mathrm{LL}_{0(1)}$ transition. Here, we observe a pronounced reduction of the decay time to $\tau=3 \pm 1$ ps, i.e., the dynamics becomes almost by one order of magnitude faster than in the case of $B=0$. This very fast decay of the pump-probe signal is surprising, since the Landau level spacing does not match the energy of optical phonons and thus the carrier-phonon scattering is expected to be strongly quenched $^{34,35}$.

To shed light on the nature of the observed fast population change responsible for the acceleration of the carrier dynamics, we employ circularly polarized radiation $\left(\sigma^{+}\right.$and $\sigma^{-}-$ radiation). We record DTS successively for all four combinations of pumping and probing with $\sigma^{+}$and $\sigma^{-}$-radiation, cf. Fig. 2a. According to the optical selection rules, $\sigma^{+}$-radiation pumps the $\mathrm{LL}_{-1} \rightarrow \mathrm{LL}_{0}$ and $\sigma^{-}$-radiation the $\mathrm{LL}_{0} \rightarrow \mathrm{LL}_{1}$ transition ${ }^{36}$, cf. Fig. $2 b$. It is instructive to visualize the expected DTS sign in the absence of scattering for intrinsic graphene, i.e. for initial occupations $\rho_{0}=0.5, \rho_{-1}=1$, and $\rho_{1}=0$. In this fully symmetric 
system assuming that the dynamics is only determined by Pauli-blocking, one expects positive DTS (increased transmission) for pumping and probing with the same polarization state and negative pump-probe signals (increased absorption) for pumping and probing with the opposite polarization state, cf. Fig. 2b. The signals within one pair of similar and opposite polarization feature similar amplitudes. For doped graphene the symmetry of the system is broken and hence, the absolute values of the induced transmission differ for the four different polarization combinations. Nevertheless, this does not change the expected DTS sign discussed above. Also energy relaxation via phonons as well as defect-mediated phonon scattering has no influence, as these processes relax the carrier distribution back into the equilibrium state.

The experiments show the following behavior: The DTS for pumping with $\sigma^{-}$-radiation (Fig. $2 \mathrm{c}$ and 2e) exhibit a fast initial decay of the order of the pulse duration and a slower relaxation component. The sign of the DTS is in accord with the expectation considering the pump scheme depicted in Fig. 2b. In particular, negative DTS is observed for pumping with $\sigma^{-}-$ radiation and probing with $\sigma^{+}$-radiation (Fig. 2e). For pumping with $\sigma^{+}$-radiation and probing with $\sigma^{-}$-radiation (Fig. 2g), however, an unexpected positive signal is observed indicating that a strong redistribution of carriers must take place, beyond the effect induced by the photon field. A possible explanation is efficient Auger scattering, which can lead to a fast redistribution of carriers giving rise to a different sign of the DTS. One can distinguish two counteracting Auger scattering processes inducing $\mathrm{LL}_{0} \rightarrow \mathrm{LL}_{-1}, \mathrm{LL}_{0} \rightarrow \mathrm{LL}_{1}$ and $\mathrm{LL}_{-1} \rightarrow \mathrm{LL}_{0}$, $\mathrm{LL}_{1} \rightarrow \mathrm{LL}_{0}$ transitions, respectively, as indicated in the sketches left of the panels containing the experimental data (cf. Fig. 2). Generally, they are referred to as Auger recombination and impact ionization, respectively. In graphene, the terminology is not straightforward, since $\mathrm{LL}_{0}$ is shared by the valence and the conduction band. Therefore, in the following, we will refer to 
$\mathrm{LL}_{0} \rightarrow \mathrm{LL}_{-1}$ and $\mathrm{LL}_{0} \rightarrow \mathrm{LL}_{1}\left(\mathrm{LL}_{-1} \rightarrow \mathrm{LL}_{0}\right.$ and $\left.\mathrm{LL}_{1} \rightarrow \mathrm{LL}_{0}\right)$ as outward (inward) Auger scattering with respect to the zeroth Landau level. Finally, the DTS for pumping with $\sigma^{+}-$ radiation and probing with $\sigma^{+}$-radiation contains a negative tail after an initially positive peak, cf. Fig. 2i. Such a behavior cannot be understood by just considering optical pumping and energy relaxation suggesting again a crucial contribution from Auger scattering.

To obtain a thorough understanding of the underlying elementary scattering processes microscopic calculations were performed. In this section, the foundation of the model is described, then, we compare experimental and calculated DTS results, and finally, we discuss the microscopic mechanism behind the experimentally observed and theoretically confirmed unexpected DTS behavior. Our model is based on the density matrix formalism ${ }^{37,38}$. Similar to previous modeling in the absence of the magnetic field ${ }^{39,18}$, we derive a set of equations describing time-resolved microscopic polarizations $\mathrm{p}_{\mathrm{nn}},(\mathrm{t})$ and population probabilities $\rho_{\mathrm{n}}(\mathrm{t})$

$$
\begin{aligned}
\dot{\rho}_{n}(t) & = \pm 2 \sum_{n^{\prime}} \operatorname{Re}\left[\Omega_{n n^{\prime}}(t) p_{n n^{\prime}}(t)\right]+S_{n}^{\text {in }}(t)\left[1-\rho_{n}(t)\right]-S_{n}^{\text {out }}(t) \rho_{n}(t) \\
\dot{p}_{n n^{\prime}}(t) & =i \triangle \omega_{n n^{\prime}} p_{n n^{\prime}}(t)+\Omega_{n n^{\prime}}(t)\left[\rho_{n}(t)-\rho_{n^{\prime}}(t)\right]-\frac{\Gamma(t)}{\hbar} p_{n n^{\prime}}(t) .
\end{aligned}
$$

Here, the magnetic field was introduced in the Dirac equation via the Peierls substitution ${ }^{36}$. The equations include the optical excitation as well as Coulomb- and phonon-induced manyparticle scattering processes. The strength of the carrier-light interaction is given by the optical matrix element and depends on the excitation field, both being incorporated into the Rabi frequency $\Omega_{\mathrm{nn}}(\mathrm{t})$. The time- and LL-dependent in- and out-scattering rates $S_{\mathrm{n}}{ }^{\text {in/out }}(\mathrm{t})=$ $\left.\mathrm{S}_{\mathrm{n}}{ }^{\text {in/out }}\right|_{\text {Coulomb }}(\mathrm{t})+\left.\mathrm{S}_{\mathrm{n}}{ }^{\text {in/out }}\right|_{\text {phonon }}(\mathrm{t})$ describe energy-conserving many-particle Coulomb processes and inelastic scattering with phonons. The efficiency of the former is determined by the Coulomb matrix elements that are obtained using tight-binding wave functions. The microscopic treatment of the Coulomb interaction is crucial for understanding the experimentally observed fast carrier dynamics. In contrast, the carrier-phonon scattering is 
negligibly small at the beginning due to the mismatch of the optical phonon energy and the investigated inter-Landau-level transitions. Scattering with acoustic phonons assisted by impurities is expected to contribute to the experimentally observed slower component in the differential transmission spectra (for details refer to methods section on the modelling). The modeling was performed for low n-type graphene with a carrier concentration of $6 \times 10^{10} \mathrm{~cm}^{-2}$ that corresponds a filling of $\mathrm{LL}_{0}$ of $64 \%$ (At $\mathrm{B}=0$ this would correspond to a Fermi energy of $28 \mathrm{meV}$, see also methods section on sample doping). First, we optically generate a nonequilibrium distribution by applying a circularly polarized pulse with a width of $2.7 \mathrm{ps}$, a pump fluence of $0.1 \mu \mathrm{J} / \mathrm{cm}^{2}$, and an excitation energy $75 \mathrm{meV}$ corresponding to the experimental realization. Then, we investigate the temporal evolution of the microscopic polarization $\mathrm{p}_{\mathrm{nn}}$, and the carrier occupations $\rho_{\mathrm{n}}(\mathrm{t})$ in the involved Landau levels. We focus on the carrier dynamics within the energetically lowest Landau levels.

The calculated differential transmission reproduces well all qualitative features of the measured DTS, cf. Fig. 2, right column. For pumping with $\sigma^{+}$-radiation, the theoretical curves show the same surprising result as observed in the experiment, namely the unexpected positive signal for probing with $\sigma^{-}$-radiation and the initially positive and subsequently negative signal for probing with $\sigma^{+}$-radiation (cf. Fig. $2 \mathrm{~h}$ and $2 \mathrm{j}$ ). We note that by artificially weakening the electron-electron coupling the expected negative DTS is obtained for pumping and probing with opposite polarization (not shown), confirming that Auger processes are indeed the reason for the surprising positive DTS observed in the experiment, as further discussed below. Note that for pumping with $\sigma^{-}$-radiation, the signals are in accord with the expectations considering the optical selection rules, however, when probing with $\sigma^{+}$-radiation a plateau after the initial peak is observed in the DTS (cf. Figs. 2f). This is an indication that also in this configuration the underlying dynamics is more complex than a simple relaxation 
to the equilibrium. The processes leading to the plateau are further discussed in the supplementary information.

Since our calculations give microscopic access to the time-resolved populations of the Landau levels, we can reveal the underlying elementary scattering processes responsible for the observed and theoretically predicted surprising features in the differential transmission spectra. Our calculations clearly reveal that Auger processes play the crucial role. They give rise to an efficient redistribution of carriers in the energetically equidistant $\mathrm{LL}_{0}, \mathrm{LL}_{1}$, and $\mathrm{LL}_{-1}$. The time-resolved occupations of the energetically lowest Landau levels are shown in Fig. 3: For pumping with $\sigma^{-}$-radiation, optical excitation depopulates $\mathrm{LL}_{0}$ leading to a decrease of $\rho_{0}(t)$ (cf. Fig. 3b). At the same time, $L_{1}$ becomes populated resulting in an increase of $\rho_{1}(t)$. In the case of pumping with $\sigma^{-}$-radiation, inward Auger scattering dominates, since it counteracts the optical pumping (cf. Fig. 3a). This effect causes the decreased occupation of $\mathrm{LL}_{-1}$, which is not affected by the optical pumping (cf. Fig. 3b). In contrast, for pumping with $\sigma^{+}$-radiation, outward Auger scattering dominates over inward processes (cf. Fig. 3c). In n-type graphene, the Auger scattering is so efficient, that after an initial increase of $\rho_{0}(t)$ due to the optical pumping an Auger-induced decrease is found cf. Fig. 3d. As a result, we do not observe enhanced absorption and negative DTS as expected for pumping with $\sigma^{+}$and probing with $\sigma^{-}$ radiation (cf. Fig. 2b), but rather a positive pump-probe signal, cf. Figs. $2 \mathrm{~g}$ and $2 \mathrm{~h}$. The Auger scattering efficiently populates $\mathrm{LL}_{1}$ giving rise to absorption bleaching of the $\sigma^{-}$-probe pulse resulting in positive differential transmission. The calculations reveal that $\rho_{-1}(t)$ quickly recovers, while the depopulated $\mathrm{LL}_{0}$ relaxes back much more slowly to its equilibrium occupation. This difference in the carrier dynamics causes the sign change of the DTS observed for pumping and probing with $\sigma^{+}$-radiation, cf. Figs. $2 \mathrm{i}$ and $2 \mathrm{j}$. 
Note that for intrinsic graphene the effect of the Auger processes on the carrier distribution is not strong enough to change the sign of the DTS. Already small doping breaks this symmetry and results in the observed surprising effects. The same effects observed for $n$-doped graphene under pumping with $\sigma^{+}$-radiation occur for $\mathrm{p}$-doped graphene under pumping with $\sigma^{-}$-radiation. A discussion how this is related to basic thermodynamic principles is provided in the supplementary material.

Our study conclusively shows that carrier-carrier scattering is the predominant mechanism for carrier redistribution in Landau-quantized graphene. Scattering with optical phonons, on the other hand, is expected to be strongly suppressed unless the LLs are resonant with the optical phonon energy ${ }^{34,35}$. In the performed experiments, we find only a moderate increase of the decay time with the increasing magnetic field from $18 \pm 3$ ps at $0 \mathrm{~T}$ to $22 \pm 3$ ps at $3 \mathrm{~T}$ (cf. Fig. $1 \mathrm{~b}$ and 1e). A possible explanation for this decay component could be defect-assisted scattering with acoustic phonons, which has been identified as an important scattering mechanism in the absence of magnetic fields ${ }^{40,41}$. The role of these relaxation channels for Landau-quantized graphene still needs to be thoroughly investigated in future studies.

In conclusion, our results show that Landau quantized graphene is ideal to investigate strong carrier-carrier scattering processes. The levels $\mathrm{LL}_{-1}, \mathrm{LL}_{0}$ and $\mathrm{LL}_{1}$ are decoupled from the remaining spectrum both with respect to optical excitation and scattering. The equidistant but optically selectively addressable three-levels are an ideal system to study energy-conserving carrier-carrier scattering processes. The possibility of polarization sensitive induced absorption and induced transmission can be applied for concepts of optical switching with high functionality. In quantum information processing, circularly polarized radiation is often used to address quantum bits. The switching in Landau quantized graphene can be controlled all-optically by the polarization state of the radiation. Electric gating allowing one to switch 
from n-type to p-type graphene would add even more functionality. Furthermore the nonequidistant Landau spectrum of graphene offers the possibility to develop a tunable laser. For example, pumping the $\mathrm{LL}_{-2} \rightarrow \mathrm{LL}_{1}$ transition should result in population inversion between $\mathrm{LL}_{1}$ and $\mathrm{LL}_{0}$ for samples with a low initial population of $\mathrm{LL}_{0}$ (p-type graphene). For realizing this scheme, detailed knowledge of Auger processes is essential, as they non-radiatively depopulate $\mathrm{LL}_{1}$ and thereby limit the performance of such a laser. On the other hand, Auger scattering may be directly exploited to realize carrier multiplication in Landau quantized graphene. To this end, pumping the $\mathrm{LL}_{-3} \rightarrow \mathrm{LL}_{4}$ transition Auger-scattering between the equidistant $\mathrm{LL}_{4}, \mathrm{LL}_{1}$ and $\mathrm{LL}_{0}$ can facilitate carrier multiplication.

\section{Methods}

\section{Pump-probe spectroscopy}

The free-electron laser FELBE provided frequency tunable Fourier-limited radiation pulses. In the experiments described in the paper a photon energy of $75 \mathrm{meV}$ was chosen. The pulse duration of the $75-\mathrm{meV}$ pulses was $2.7 \mathrm{ps}$ (repetition rate $13 \mathrm{MHz}$ ). The pulses were split into pump and probe pulses by a pellicle beam splitter. The polarization of pump and probe beam were controlled independently. Frequency-tunable quarter-wave plates (from Alphalas $\mathrm{GmbH}$ ) were used for generating circularly polarized radiation. Both the pump and probe beam were focused on the sample in the magnet cryostat by an off-axis parabolic mirror (effective focal length: $178 \mathrm{~mm}$ ). The spot size on the sample was $\sim 0.5 \mathrm{~mm}$ (full width at half maximum). The pump fluence was $\sim 0.1 \mu \mathrm{J} / \mathrm{cm}^{2}$, in this range the DTS increased linearly with pump fluence. The fluence of the probe beam was about $10 \%$ of the pump fluence. The time delay between pump and probe pulses was varied by a mechanical delay stage. 


\section{Microscopic modelling}

In this study, we focus on the impact of Coulomb interaction and include carrier-phonon scattering on a phenomenological level. To this end, the full scattering rate $\left.S_{n}{ }^{\text {in/out }}\right|_{\text {phonon }}$ including Pauli-blocking terms and phonon occupations is considered, while the corresponding electron-phonon matrix elements are adjusted to the experimentally observed decay. The phonons are assumed to be in equilibrium with a phonon bath and their occupations are described by the Bose-Einstein distribution. In contrast, the Coulomb interaction is entirely considered on a microscopic footing. We also take dynamical screening of the Coulomb potential into account by evaluating the dielectric function $\varepsilon(\mathrm{q}, \omega)$ in the random phase approximation following the approach of Ref. 36,42. The Landau level broadening is calculated self-consistently taking into account scattering of electrons on an impurity potential with a Gaussian white noise distribution ${ }^{43}$. This electron-impurity scattering yields the dominant contribution to the dephasing $\Gamma(t)$ of the microscopic polarization, which also comprises contributions of Coulomb- and phonon-induced manyparticle scattering.

\section{Sample doping}

MEG samples grown on the C-terminated face of $\mathrm{SiC}$ consist of a number of layers that are rotated against each other. Charge transfer from the $\mathrm{SiC}$ substrate results in high doping of the first layer at the interface with carrier concentration of $10^{13} \mathrm{~cm}^{-2}$. Successive layers exhibit a doping of $\sim 30 \%$ of the previous layer, hence after five layers low carrier concentrations in the range of $10^{10} \mathrm{~cm}^{-2}-10^{11} \mathrm{~cm}^{-2}$ are reached ${ }^{16,30}$. Pump-probe experiments, cw magnetospectroscopy and angle-resolved photoemission spectroscopy indicate that a substantial number of layers exhibits doping in the range of $10^{10} \mathrm{~cm}^{-2} 9,18,44$. For the sample used in our experiment layers with carrier concentrations of $\sim 10^{10} \mathrm{~cm}^{-2}$ were evidenced by magnetospectroscopy and pump-probe experiments. The experiments in all four combinations of 
pumping and probing with $\sigma^{+}$and $\sigma^{-}$-radiation in magnetic fields directly indicate that $\mathrm{LL}_{0}$ can neither be completely filled nor completely empty. The completely filled zeroth LL is populated with of $2 \times 10^{11} \mathrm{~cm}^{-2}$ electrons. For $\mathrm{B}=0$ this corresponds to a Fermi energy of $53 \mathrm{meV}$. Note that intrinsic graphene is characterized by $50 \%$ filling of $\mathrm{LL}_{0}$. For the microscopic modelling a value of $6 \times 10^{10} \mathrm{~cm}^{-2}$ was used. This value, being in between the experimentally determined borders, provides an overall good agreement between experimental and calculated DTS. By varying the carrier concentration in the calculation, individual features of the DTS change slightly, however, the surprising DTS sign change remains. The experimental signals contain contributions from graphene of different doping due to the gradient in doping towards the substrate. Furthermore, there are slight lateral inhomogeneities that are averaged by the applied infrared beams with a diameter of $\sim 0.5 \mathrm{~mm}$.

\section{Acknowledgements}

Support from the German Science Foundation DFG in the framework of the Priority Program 1459 Graphene. E. M. is thankful to the Einstein Foundation Berlin. The research at the freeelectron laser FELBE was supported by the European Community's Seventh Framework Programme (FP7/2007-2013) under Grant agreement No. 226716. Part of this work has been supported by ERC-2012-AdG-320590 MOMB project as well as the EC graphene flagship. We are grateful to P. Michel and the FELBE team for their dedicated support.

\section{Author contributions}

S.W., M.M., M.H., M.O. and M.P. conceived the experiments; M.M. performed the experiments, partly together with M.O. and S.W.; M.M., S.W., H.S. and M.H. analyzed and interpreted the data; F.W., E.M. and A.K. developed the microscopic theoretical model. M.O. and M.P. originated the considerations for critical level occupation described in the supplementary material. C.B. and W.A.d.H. prepared the sample; S.W. and E.M. wrote the 
paper with major input and edits from M.H. All authors discussed the results and commented on the manuscript.

Correspondence and requests for materials should be addressed to M.M. or S.W.

\section{Competing financial interests}

The authors declare no competing financial interests. 


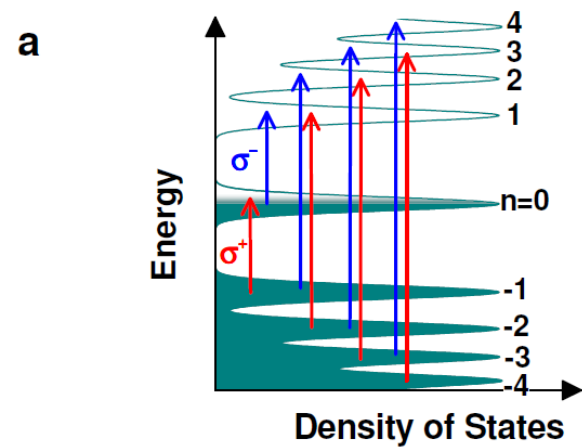

b

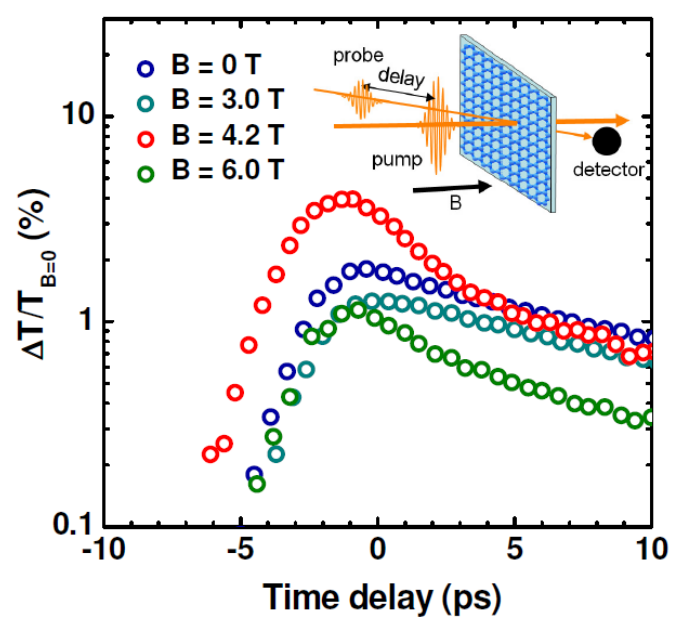

Magnetic field (T)

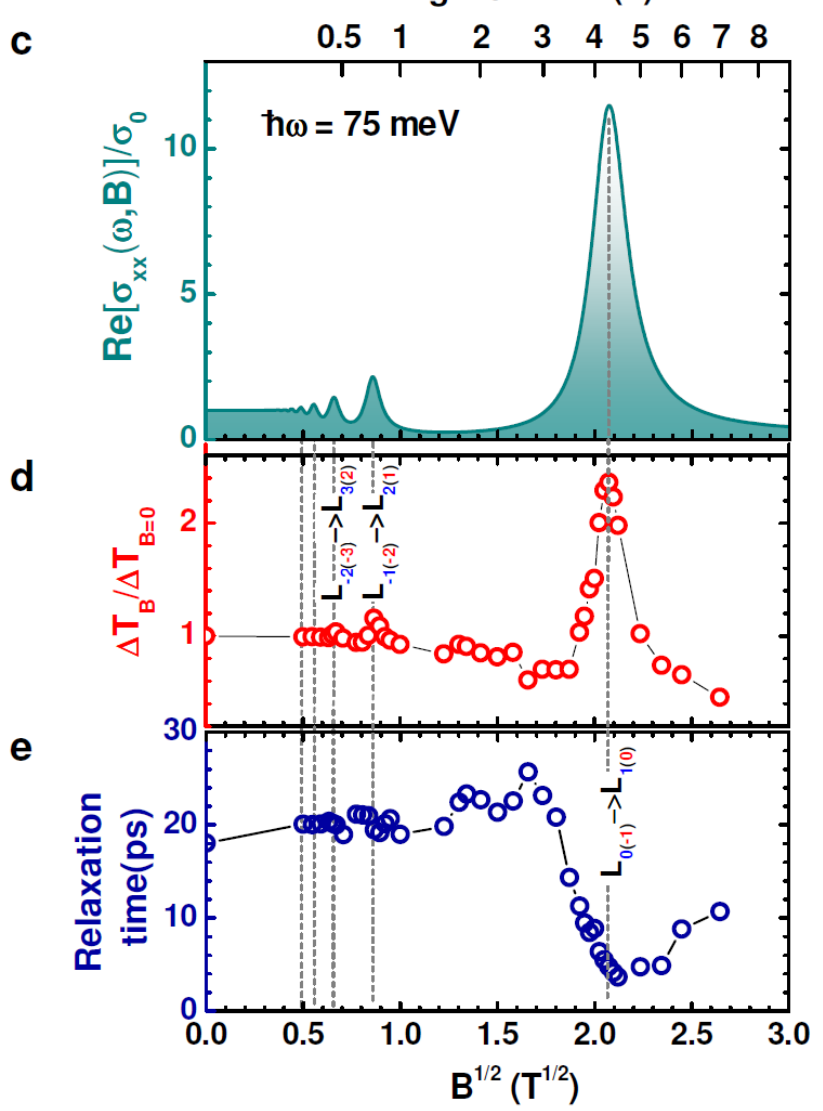

Figure 1: Pump-probe spectroscopy on graphene with linearly polarized radiation. a, Landau level spectrum of graphene with allowed dipole transitions for $\sigma^{-}$and $\sigma^{+}$-radiation. $\mathbf{b}$, Pump-probe signals for different magnetic fields. In the inset the pump-probe experiment involving linearly polarized beams is depicted. In this case, transitions allowed for $\sigma^{-}-$ radiation as well as $\sigma^{+}$-radiation are excited and probed. $\mathbf{c}$, The dynamic conductivity, which is proportional to the absorption, of one graphene layer. The calculation, as well as all experimental data in the other panels, corresponds to a photon energy of $75 \mathrm{meV}$. d, Bdependent pump-induced maximum transmission change normalized to the case at zero magnetic field. e, Initial decay time of the pump-probe signal for varied magnetic fields. 

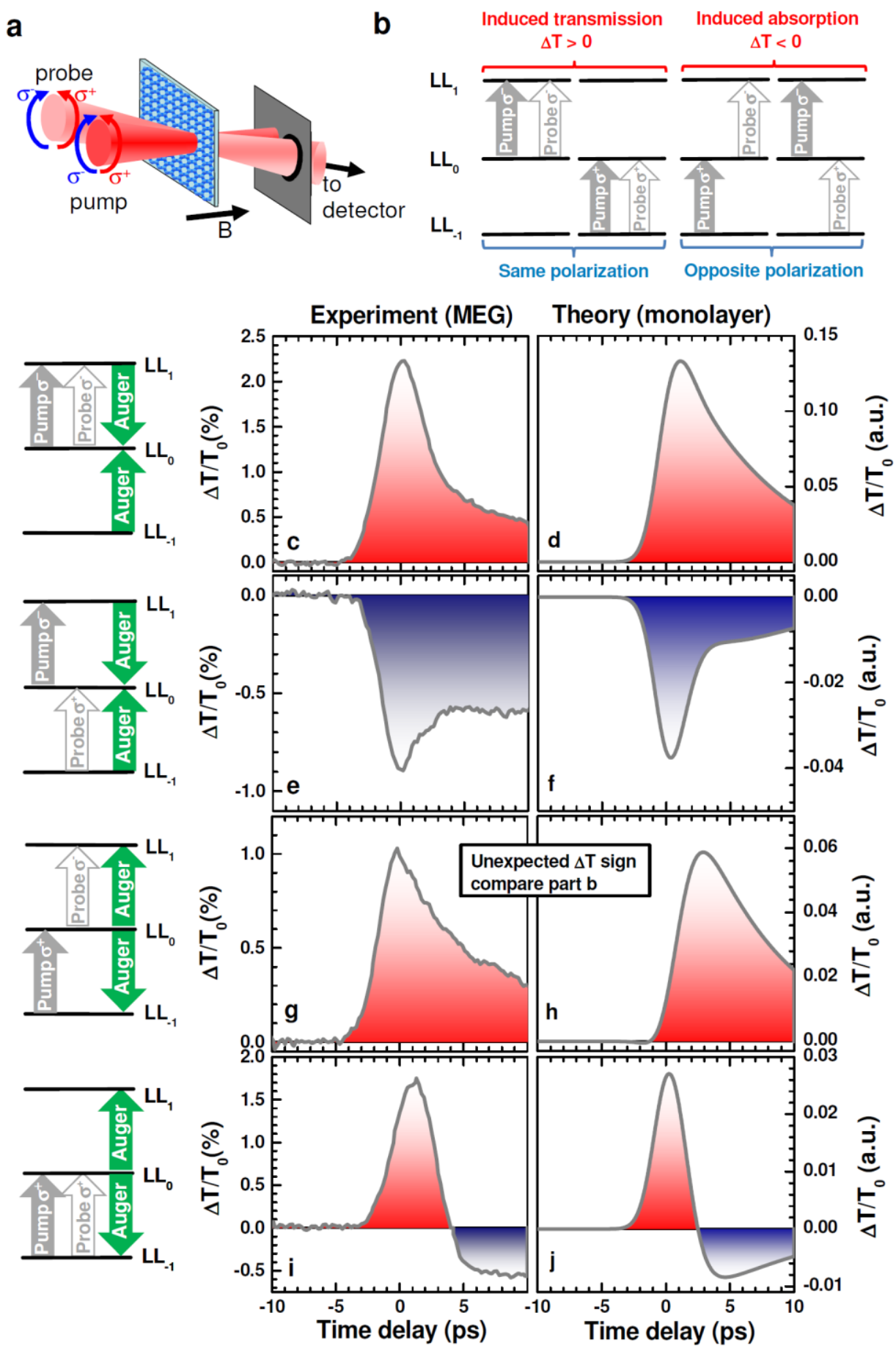

Figure 2: Pump-probe spectroscopy on graphene with circularly polarized radiation. a, Configuration of the experiments for pumping and probing with $\sigma^{-}$and $\sigma^{+}$-radiation. $\mathbf{b}$, Pumping and probing with same polarization results in induced transmission, while applying opposite polarization results in induced absorption. c, e, g, i, Experimental pump-probe signals for all four combinations of pumping and probing with $\sigma^{-}$and $\sigma^{+}$-radiation. Blue and red shaded regions highlight positive and negative DTS, respectively. $\mathbf{d}, \mathbf{f}, \mathbf{h}, \mathbf{j}$, Differential transmission calculated by microscopic theory for all four combinations of pumping and probing with $\sigma^{-}$and $\sigma^{+}$-radiation. The diagrams left of the panels indicate the polarization of pump and probe beam as well as the dominating Auger scattering process, i.e. either inward or outward Auger scattering. 
a

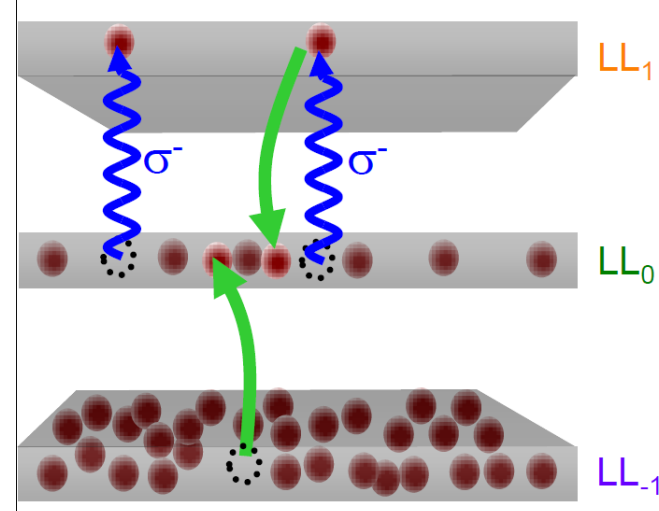

pump $\sigma^{-}$

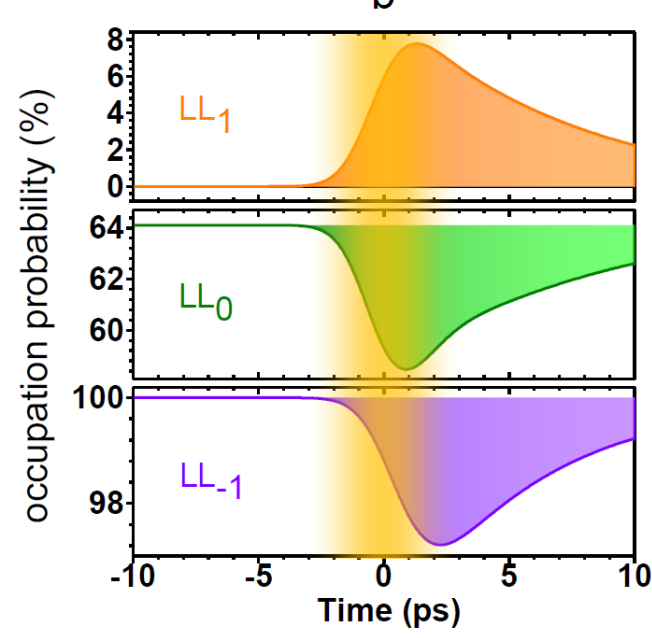

c

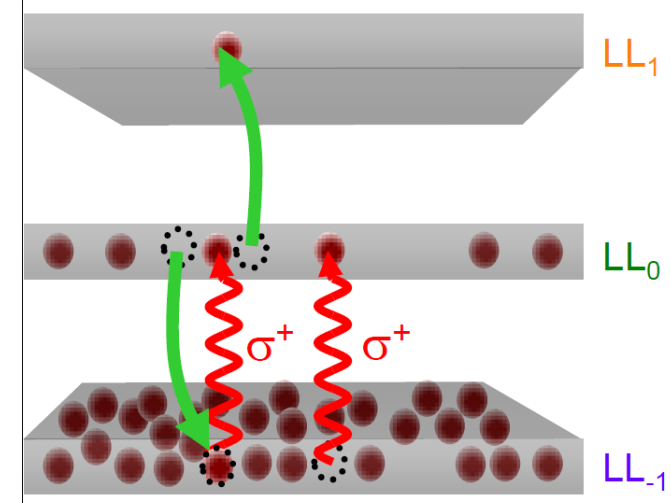

d

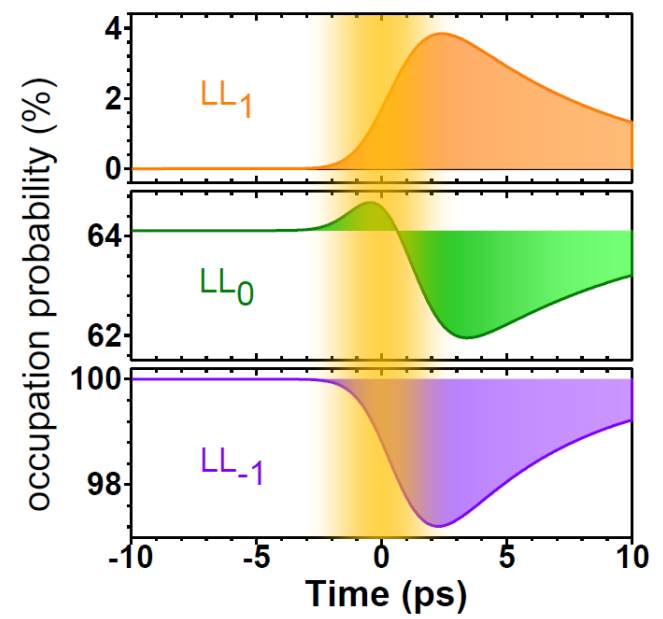

Figure 3: Level occupation for pumping with circularly polarized radiation. a, c, Illustration of the transitions between $\mathrm{LL}_{-1}, \mathrm{LL}_{0}$ and $\mathrm{LL}_{1}$ induced by optical pumping and the net Auger scattering. The green arrows symbolize the predominant contribution of inward (a) or outward (c) Auger scattering. b, d, Occupations of the Landau levels calculated by microscopic modeling taking into account the optical excitation, carrier-carrier, and carrierphonon scattering. The orange shaded area indicates the duration of the pump pulse. 


\section{References:}

[1] Drexler, C., Tarasenko, S. A., Olbrich, P., Karch, J., Hirmer, M., Müller, F., Gmitra, M., Fabian, J., Yakimova, R., Lara-Avila, S., Kubatkin, S., Wang, M., Vajtai, R., Ajayan, P. M., Kono, J. \& Ganichev, S. D. Magnetic quantum ratchet effect in graphene. Nature Nanotechnology 8, 104-107 (2013).

[2] Ponomarenko, L. A.), Gorbachev, R. V., Yu, G. L., Elias, D. C., Jalil, R., Patel, A. A., Mishchenko, A., Mayorov, A. S., Woods, C. R., Wallbank, J. R., Mucha-Kruczynski, M., Piot, B. A., Potemski, M., Grigorieva, I. V., Novoselov, K. S., Guinea, F., Fal'ko, V. I. \& Geim, A. K. Cloning of Dirac fermions in graphene superlattices. Nature 497, 594-597 (2013).

[3] Dean, C. R., Wang, L., Maher, P., Forsythe, C., Ghahari, F., Gao, Y., Katoch, J., Ishigami, M., Moon, P., Koshino, M., Taniguchi, T., Watanabe, K., Shepard, K. L., Hone, J. \& Kim, P. Hofstadter's butterfly and the fractal quantum Hall effect in moire superlattices. Nature 497, 598-602 (2013).

[4] Hunt, B., Sanchez-Yamagishi, J. D., Young, A. F., Yankowitz, M., LeRoy, B. J., Watanabe, K., Taniguchi, T., Moon, P., Koshino, M., Jarillo-Herrero, P. \& Ashoori, R. C. Massive Dirac fermions and Hofstadter butterfly in a van der Waals heterostructure. Science 340 1427-1430 (2013).

[5] Bolotin, K. I., Ghahari, F., Shulman, M. D., Stormer, H. L. \& Kim, P. Observation of the fractional quantum Hall effect in graphene. Nature 462, 196-199 (2009).

[6] Du, X. Skachko, I., Duerr, F., Luican, A. \& Andrei, E. Y. Fractional quantum Hall effect and insulating phase of Dirac electrons in graphene. Nature 462, 192-195 (2009).

[7] Novoselov, K. S., Geim, A. K., Morozov, S. V., Jiang, D., Katsnelson, M. I., Grigorieva, Dubonos, S. V. \& Firsov A. A. Two-dimensional gas of massless Dirac fermions in graphene. Nature 438, 197-200 (2005).

[8] Zhang, Y., Tan, J. W., Stormer, H. L. \& Kim, P. Experimental observation of the quantum Hall effect and Berry's phase in graphene. Nature 438, 201-204 (2005).

[9] Sadowski, M. L., Martinez, G., Potemski, M., Berger, C. \& de Heer, W. A. Landau level spectroscopy of ultrathin graphene layers. Phys. Rev. Lett. 97, 266405 (2006).

[10] Plochocka, P., Faugeras, C., Orlita, M., Sadowski, M. L., Martinez, G., Potemski, M., Goerbig, M. O., Fuchs, Berger, C. \& de Heer, W. A. High energy limit of massless Dirac fermions in multilayer graphene using magneto-optical transmission spectroscopy. Phys. Rev. Lett. 100, 087401 (2008).

[11] Orlita, M., Faugeras, C., Neugebauer, P., Martinez, Maude, D. K., Barra, A.-L., Sprinkle, M., Berger, C., de Heer, W. A. \& Potemski, M. Approaching the Dirac point in high-mobility multilayer epitaxial graphene. Phys. Rev. Lett. 101, 267601 (2008).

[12] Neugebauer, P., Orlita, M., Faugeras, C., Barra, A.-L. \& Potemski, M. How perfect can graphene be? Phys. Rev. Lett. 103, 136403 (2009).

[13] Crassee, I, Levallois, J., Walter, A. L., Ostler, M., Bostwick, A., Rotenberg, E., Seyller, T., van der Marel, D. \& Kuzmenko, A. B. Giant Faraday rotation in single- and multilayer graphene. Nature Phys. 7, 48-51 (2011).

[14] Kawano, Y. Wide-band frequency tunable terahertz and infrared detection with graphene. Nanotech. 24, 21404 (2013).

[15] Dawlaty, J. M., Shivaraman, S., Chandrashekhar, M., Rana, F., \& Spencer M. G. Measurement of ultrafast carrier dynamics in epitaxial graphene. Appl. Phys. Lett. 92, 042116 (2008).

[16] Sun, D., Wu, K., Divin, C., Li, X., Berger, C., de Heer, W. A., First, P. N. \& Norris, T. B. Phys. Rev. Lett. 1011574 (2008). 
[17] Breusing, M., Kuehn, S., Winzer, T., Malic, E, Milde, F., Severin, N., Rabe, J. P., Ropers, C., Knorr, A., \& Elsaesser, T. Ultrafast nonequilibrium carrier dynamics in a single graphene layer. Phys. Rev. B $\mathbf{8 3}$ 153410 (2011).

[18] Winnerl, S., Orlita, M., Plochocka, P., Kossacki, P., Potemski, M., Winzer, T., Malic, E., Knorr, A., Sprinkle, M., Berger, C., de Heer, W. A., Schneider, H. \& Helm M. Carrier relaxation in epitaxial graphene photoexcited near the Dirac point. Phys. Rev. Lett. 107, 237401 (2011).

[19] Brida, D., Tomadin, A., Manzoni, C., Kim, Y. J., Lombardo, Milana, S., Nair, R. R., Novoselov, K. S., Ferrari, A. C., Cerullo, G. \& Polini, M. Ultrafast collinear scattering and carrier multiplication in graphene. Nature Commun. 4, 1987 (2013).

[20] Tielrooij, K. J., Song, J. C. W., Jensen, S. A., Centeno, A., Pesquera, A., Elorza, A. Z., Bonn, M., Levitov, L. S. \& Koppens, F. H. L. Photoexcitation cascade and multiple hot-carrier generation in graphene. Nature Phys. 9, 248-252 (2013).

[21] Plochocka, P., Kossacki, P., Golnik, A., Kazimierczuk, T., Berger, C., de Heer, W. A. \& Potemski, M. Slowing hot-carrier relaxation in graphene using a magnetic field. Phys. Rev. B 80, 245415 (2009).

[22] Foster, M. S. \& Aleiner, I. L. Slow imbalance relaxation and thermoelectric transport in graphene. Quasiclassical cyclotron resonance of Dirac fermions in highly doped graphene. Phys. Rev. B 79, 085415 (2010).

[23] Otsuji, T., Boubanga Tombet, S. A., Satou, A., Fukidome, H., Suemitsu, M., Sano, E., Popov, V., Ryzhii, M. \& Ryzhii, V. Graphene-based devices in terahertz science and technology. J. Phys. D: Appl. Phys. 45, 303001 (2012).

[24] Rana, F. Electron-hole generation and recombination rates for Coulomb scattering in graphene. Phys. Rev. $B$ 76, 155431 (2007).

[25] Winzer, T., Knorr, A. \& Malic, E. Carrier multiplication in graphene, Nano Lett. 10, 4839-4843 (2010).

[26] Winzer, T. \& Malic, E., Impact of Auger processes on carrier dynamics in graphene. Phys. Rev. B 85, 241404 (2012).

[27] Tani, S., Blachard, F. \& Tanaka, K. Ultrafast carrier dynamics in graphene under a high electric field. Phys. Rev. Lett. 109, 166603 (2012).

[28] Berger, C.; Song, Z.; Li, X.; Wu, X.; Brown, N.; Naud, C.; Mayou, D.; Li, T.; Hass, J; Marchenkov, A. N.; Conrad, E. H.; First, P. N.; de heer, W. A. Electronic confinement and coherence in patterned epitaxial graphene. Science 312, 1191-1196 (2006)

[29] Ferrari, A. C., Meyer, J. C., Scardaci, V., Casiraghi, C., Lazzeri, M., Mauri, F., Piscanec, S., Jiang, D., Novoselov, K. S., Roth, S. \& Geim, A. K. Raman spectrum of graphene and graphene layers. Phys. Rev. Lett. 97, 187401 (2006).

[30] Sun, D., Divin, C., Berger, C., de Heer, W. A., First, P. N. \& Norris, T. B. Spectroscopic measurement of interlayer screening in multilayer epitaxial graphene. Phys. Rev. Lett. 104136802 (2010).

[31] Winnerl, S., Göttfert, F., Mittendorff, M., Schneider, H., Helm, M., Winzer, T., Malic, E., Knorr, A., Orlita, M., Potemski, M., Sprinkle, M., Berger, C., \& de Heer, W. A. Time-resolved spectroscopy on epitaxial graphene in the infrared spectral range: relaxation dynamics and saturation behavior. J. Phys.: Condens. Matter 25, 054202 (2013).

[32] Witowski, A. M., Orlita, M., Stepniewski, R., Wysmołek, A., Baranowski, J. M., Strupiński, W., Faugeras, C., Martinez, G. \& Potemski M. Quasiclassical cyclotron resonance of Dirac fermions in highly doped graphene. Phys. Rev. B 82, 165305 (2010). 
[33] Orlita M., Crassee, I., Faugeras, C., Kuzmenko, A. B., Fromm, F., Ostler, M., Seyller, Th., Martinez, G., Polini, M. \& Potemski, M. Classical to quantum crossover of the cyclotron resonance in graphene: a study of the strength of intraband absorption. New J. Phys. 14, 095008 (2012).

[34] Wang, Z.-W., Liu, L., Shi, L., Gong, X.-J., Li, W.-P. \& Xu, K. The temperature dependence of optical phonon scattering in graphene under strong magnetic field. J. Phys. Soc. Jpn. 82, 094606 (2013).

[35] Wendler, F., Knorr, A. \& Malic, E. Resonant carrier-phonon scattering in graphene under Landau quantization. Appl. Phys. Lett. 103, 253117 (2013)

[36] Goerbig, M. O. Electronic properties of graphene in a strong magnetic field, Rev. Mod. Phys. 83, 1193 (2011).

[37] Haug, H. \& Koch, S. W. Quantum Theory of the Optical and Electronic Properties of Semiconductors, World Scientific (2009)

[38] Malic, E. \& Knorr, A. Graphene and Carbon Nanotubes - Ultrafast Relaxation Dynamics and Optics, Wiley-VCH

[39] Malic, E., Winzer, T., Bobkin, E. \& Knorr, A. Microscopic theory of absorption and ultrafast manyparticle kinetics in graphene. Phys. Rev. B, 84, 205406 (2011)

[40] Graham, M. W., Shi, S. F., Ralph, D. C., Park, J. \& McEuen, P. L. Photocurrent measurements of supercollision cooling in graphene. Nature Phys. 9, 103-108 (2013).

[41] Betz, A. C., Jhang, S. H., Pallecchi, E., Ferreira, R., Feve, G., Berroir, J. M. \& Placais, B. Supercollision cooling in undoped graphene, Nature Phys. 9, 109-112 (2013).

[42] Roldán, R., Goerbig, M. O. \& Fuchs, J.-N. The magnetic field particle-hole excitation spectrum in doped graphene and in a standard two-dimensional electron gas. Semicond. Sci. Technol. 25, 034005 (2010).

[43] Ando, T. \& Uemura, Y., Theory of quantum transport in a two-dimensional electron system under magnetic fields. I. Characteristics of level broadening and transport under strong fields. J. Phys. Soc. Jpn. 36, 959-967 (1974).

[44] Sprinkle, M., Siegel, D. Hu, Y., Tejeda, A., Teleb-Ibrahimi, A., Le Fevre, P., Bertram, F., Vizzini, S., Enriquez, H., Chiang, S., Soukiassian, P., Berger, C., de Heer., W.A., Lanzara, A., \& Conrad, E.H. First direct observation of a nearly ideal graphene band structure, Phys. Rev. Lett. 103, 226803 (2009). 